\title{
Preface
}

\section{Special issue: ICNC-FSKD 2015}

\author{
Zheng Xiao and Kenli $\mathrm{Li}^{*}$ \\ Hunan University, China
}

This special issue of the Journal of Intelligent and Fuzzy Systems (JIFS) publishes a selection of excellent papers presented at the 11th International Conference on Natural Computation (ICNC'15) and the 12th International Conference on Fuzzy Systems and Knowledge Discovery (FSKD'15), jointly held during August 15-17, 2015 in Zhangjiajie, China.

ICNC-FSKD is a premier international forum for scientists and researchers to present the state of the art on data mining and intelligent methods inspired from nature, particularly biological, linguistic, and physical systems, with applications to computers, circuits, systems, control, communications, and more.

ICNC-FSKD 2015 received 1144 manuscripts in total from over 40 different countries. More than 500 people from universities, institutes, and companies worldwide attended the conference. Through a two round peer to peer review process, 42 papers were selected for this special issue after patiently extending and revising manuscripts to meet the comments and suggestions of the referees and responding to meet the quality standards of the journal.

The topics covered in this special issue include: Fuzzy theory and its applications, Artificial Intelligence Algorithms, Intelligent Information Processing, High Performance Computing, and Information Systems. It is hoped that this JIFS issue will make a good reference material and be of great use for readers in Mathematics, Computer Science as well as System Engineering amongst others.

Kenli Li

Chair of ICNC-FSKD 2015

E-mail:1kl@hnu.edu.cn

Guest Editors: Zheng Xiao and Kenli Li

${ }^{*}$ Corresponding author. Kenli Li, Hunan University, China. E-mail: 1k1@hnu.edu.cn. 